\title{
Finerenone Dose-Exposure-Response for the Primary Kidney Outcome in FIDELIO-DKD Phase III: Population Pharmacokinetic and Time-to-Event Analysis
}

\author{
Paul van den Berg ${ }^{1} \cdot$ Martijn Ruppert ${ }^{1} \cdot$ Emir Mesic $^{1} \cdot$ Nelleke Snelder $^{1} \cdot$ Andreas Seelmann $^{2} \cdot$ Roland Heinig $^{3}$. \\ Amer Joseph ${ }^{4} \cdot$ Dirk Garmann $^{2} \cdot$ Joerg Lippert ${ }^{2} \cdot$ Thomas Eissing $^{2}$ (1)
}

Accepted: 8 October 2021 / Published online: 13 November 2021

(c) The Author(s) 2021

\begin{abstract}
Background Finerenone is a nonsteroidal selective mineralocorticoid receptor antagonist that recently demonstrated efficacy in delaying chronic kidney disease progression and reducing cardiovascular events in patients with chronic kidney disease and type 2 diabetes in FIDELIO-DKD, where 5734 patients were randomized 1:1 to receive either titrated finerenone doses of 10 or $20 \mathrm{mg}$ once daily or placebo, with a median follow-up of 2.6 years.

Methods Nonlinear mixed-effects population pharmacokinetic models were used to analyze the pharmacokinetics in FIDELIO-DKD, sparsely sampled in all subjects receiving finerenone. Post-hoc model parameter estimates together with dosing histories allowed the computation of individual exposures used in subsequent parametric time-to-event analyses of the primary kidney outcome.

Results The population pharmacokinetic model adequately captured the typical pharmacokinetics of finerenone and its variability. Either covariate effects or multivariate forward-simulations in subgroups of interest were contained within the equivalence range of $80-125 \%$ around typical exposure. The exposure-response relationship was characterized by a maximum effect model estimating a low half-maximal effect concentration at $0.166 \mu \mathrm{g} / \mathrm{L}$ and a maximal hazard decrease at $36.1 \%$. Prognostic factors for the treatment-independent chronic kidney disease progression risk included a low estimated glomerular filtration rate and a high urine-to-creatinine ratio increasing the risk, while concomitant sodium-glucose transport protein 2 inhibitor use decreased the risk. Importantly, no sodium-glucose transport protein 2 inhibitor co-medication-related modification of the finerenone treatment effect per se could be identified.

Conclusions None of the tested pharmacokinetic covariates had clinical relevance in FIDELIO-DKD. Finerenone effects on kidney outcomes approached saturation towards $20 \mathrm{mg}$ once daily and sodium-glucose transport protein 2 inhibitor use provided additive benefits.
\end{abstract}

\section{Introduction}

Chronic kidney disease (CKD) in patients with type 2 diabetes (T2D) is associated with an accelerated progression

Thomas Eissing

thomas.eissing@bayer.com

1 Leiden Experts on Advanced Pharmacokinetics and Pharmacodynamics (LAP\&P), Leiden, The Netherlands

2 Pharmacometrics, Pharmaceuticals R\&D, Bayer AG, Leverkusen, Germany

3 Clinical Pharmacology, Pharmaceuticals R\&D, Bayer AG, Wuppertal, Germany

4 Clinical Development, Pharmaceuticals R\&D, Bayer AG, Berlin, Germany towards kidney failure and a high burden of cardiovascular mortality and morbidity [1]. The FIDELIO-DKD phase III study (ClinicalTrials.gov number, NCT02540993) investigated the efficacy and safety of the nonsteroidal, selective mineralocorticoid receptor antagonist (MRA) finerenone compared to placebo in patients with CKD and T2D [2]. In total, 5734 patients were randomized 1:1 to receive finerenone ( 10 or $20 \mathrm{mg}$ once daily) or placebo and the median duration of follow-up was 2.6 years, making it one of the largest contemporary studies to evaluate patients with CKD and T2D. Dosing and titration were based on potassium and estimated glomerular filtration rate (eGFR). The primary outcome of the study was that finerenone significantly delayed the progression of kidney disease compared with placebo based on a time-to-event (TTE) analysis of the primary composite kidney endpoint 


\section{Key Points}

Finerenone is a nonsteroidal, selective mineralocorticoid receptor antagonist that recently demonstrated efficacy in delaying chronic kidney disease (CKD) progression and reducing cardiovascular events in patients with CKD and type 2 diabetes in the pivotal FIDELIO-DKD study.

In this secondary model-based analysis, sparse pharmacokinetic samples taken in all subjects taking finerenone revealed no clinically relevant pharmacokinetic covariates in FIDELIO-DKD and allowed the estimation of individual exposures as a basis for the investigation of the dose-exposure response relationship for the primary kidney outcome.

Finerenone effects saturated towards $20 \mathrm{mg}$ once daily supporting the studied dosing regimen.

Multivariate effects of established CKD markers were quantified and indicated independent and additive benefits of sodium-glucose transport protein 2 inhibitors on top of finerenone.

[3]. As a key secondary outcome of the study, finerenone also significantly reduced the risk of the composite cardiovascular endpoint [3, 4]. FIDELIO-DKD results have also been further discussed, for example, [5, 6].

Finerenone is a non-steroidal MRA that selectively blocks the mineralocorticoid receptor. The mode of action of finerenone is primarily through organ protection by counteracting inflammatory and fibrotic processes, including in the kidneys and heart, mediated by overactivation of the mineralocorticoid receptor and aldosterone upregulation $[1,7,8]$.

Besides FIDELIO-DKD, the efficacy and safety of finerenone are investigated in three additional phase III studies: FIGARO-DKD (NCT02545049), which recently completed [9] in patients with less advanced CKD and T2D compared with patients in FIDELIO-DKD, FIND-CKD (NCT05047263) in patients with non-diabetic CKD, and FINEARTS-HF (NCT04435626) in patients with heart failure with a left ventricular ejection fraction of $\geq 40 \%$, whereby FIGARO-DKD and FINARTS-HF have cardiovascular outcomes as primary endpoints.

The clinical pharmacology program for finerenone comprises 27 phase I studies to date and the main results have been published [10-15]. The program was complemented by population pharmacokinetic (PK) and pharmacodynamic analyses including evaluations of patients in latestage studies. Population PK and pharmacodynamic analyses of data from the phase IIb studies ARTS-DN (global: NCT01874431 and Japan: NCT01968668) have been published [16-18]. Here, the ARTS-DN population pharmacokinetic and pharmacodynamic analyses [17] have been extended to the pivotal phase III study FIDELIO-DKD to include an extensive covariates analysis for pharmacokinetics also providing post-hoc parameter estimates to calculate exposures for a subsequent exposure-response (ER) analysis of which the ER analysis for the primary kidney composite TTE endpoint including an evaluation of prognostic factors is described.

\section{Methods}

\subsection{Clinical Study}

Details on study design, patient characteristics, and the main results have been published [2-4]. Information on informed consent, ethics, and analytical methods are summarized in the Electronic Supplementary Material (ESM).

In brief, FIDELIO-DKD was a randomized, double-blind, placebo-controlled phase III study investigating the efficacy and safety of finerenone, in addition to standard of care with maximally tolerated labeled doses of a renin-angiotensin system inhibitor, on the progression of kidney disease in patients with T2D with CKD. The starting dose of the study drug was selected based on eGFR measured at the screening visit: patients started on finerenone $10 \mathrm{mg} /$ day or matching placebo if their eGFR was between 25 and $<60 \mathrm{~mL} /$ $\mathrm{min} / 1.73 \mathrm{~m}^{2}$; if their eGFR was $\geq 60 \mathrm{~mL} / \mathrm{min} / 1.73 \mathrm{~m}^{2}$, the starting dose was $20 \mathrm{mg} /$ day. Patients could be up-titrated or down-titrated according to changes in eGFR and serum potassium and at the discretion of the investigator $[2,19]$.

Plasma concentrations of finerenone were determined using a sparse sampling approach in all participating patients, informed via trial simulation prior to the study based on the phase IIb model to provide reliable individual exposure estimates [17]. Samples were collected at month 4 (trough sample) and at each of the yearly visits (post-dose at any time on the visit day). The primary endpoint of FIDELIO-DKD was a composite of (1) time to the first occurrence of kidney failure, (2) a sustained decrease of eGFR $\geq 40 \%$ from baseline over at least 4 weeks, or (3) renal death.

\subsection{Population PK Model Development}

The population PK model based on phase IIb data could adequately capture finerenone concentration-time data following doses from 1.25 to $20 \mathrm{mg}$ [17] and was used as a starting point for modeling PK data from FIDELIO-DKD including outlier identification as detailed in the ESM. The PK model was developed using a dataset based on an interim analysis that was planned per protocol when approximately 
two-thirds of the expected events occurred. The optimization process was focused on re-estimation of the parameters without structural model changes, re-evaluation of the random-effects structure, and a dedicated covariate analysis. The algorithm for the covariate analysis included single addition (statistical significance at the 0.01 level), forward inclusion (significance at the 0.01 level), and backward deletion (significance at the 0.001 level) steps. An overview of the tested covariates is provided in Table 1 . Upon availability of the final data, final model parameters were re-estimated.
To assess the magnitude of covariate effects, two types of model-based simulations were performed:

1. Simulations to assess the magnitude and uncertainty of the pure covariate effects.

2. Simulations to compare the exposure of finerenone at steady state in subgroups of interest taking combined (multivariate) individual covariate effects into account.

Further details on the simulations are provided in the ESM.
Table 1 Overview of tested covariates and PFs

\begin{tabular}{|c|c|c|}
\hline Covariate/PF & $\begin{array}{l}\text { Tested in finerenone } \\
\text { PK model }\end{array}$ & $\begin{array}{l}\text { Tested in TTE model for } \\
\text { renal composite endpoint }\end{array}$ \\
\hline Albumin & $X^{b}$ & \\
\hline Alcohol consumption & $X^{b}$ & $\mathrm{X}$ \\
\hline Age & $X^{a}$ & $\mathrm{X}$ \\
\hline Alkaline phosphatase & $X^{\mathrm{a}}$ & \\
\hline Alanine aminotransferase & $X^{b}$ & \\
\hline Aspartate aminotransferase & $X^{b}$ & \\
\hline Bilirubin & $X^{b}$ & \\
\hline Body height & $X^{a}$ & \\
\hline Body mass index & $\mathrm{X}^{\mathrm{a}}$ & $\mathrm{X}$ \\
\hline Body surface area & $\mathrm{X}^{\mathrm{a}}$ & \\
\hline Body weight & $\mathrm{X}^{\mathrm{a}}$ & $\mathrm{X}$ \\
\hline Lean body mass & $\mathrm{X}^{\mathrm{a}}$ & \\
\hline Likely Child-Pugh score at screening & $X^{a}$ & $\mathrm{X}$ \\
\hline Long-term co-medication of CYP3A4 inducers & $X^{a}$ & $\mathrm{X}$ \\
\hline Chronic co-medication of CYP3A4 inhibitors & $X^{a}$ & $\mathrm{X}$ \\
\hline eGFR-MDRD at baseline & $\mathrm{X}^{\mathrm{a}}$ & \\
\hline eGFR-MDRD time dependent & $\mathrm{X}^{\mathrm{a}}$ & \\
\hline eGFR-EPI at baseline & $X^{a}$ & $\mathrm{X}$ \\
\hline eGFR-EPI time dependent & $X^{a}$ & \\
\hline Ethnic group & $\mathrm{X}^{\mathrm{a}}$ & $\mathrm{X}$ \\
\hline Gamma glutamyl transferase & $X^{a}$ & \\
\hline Gender & $X^{a}$ & $\mathrm{X}$ \\
\hline GLP-1 agonist long-term co-medication & & $\mathrm{X}$ \\
\hline $\mathrm{HbA1c}$ & & $\mathrm{X}$ \\
\hline $\mathrm{K}+$ & & $\mathrm{X}$ \\
\hline Race/ethnicity & $X^{a}$ & $\mathrm{X}$ \\
\hline Serum creatinine & $X^{\mathrm{a}}$ & \\
\hline SGLT2 inhibitor chronic co-medication & $\mathrm{X}^{\mathrm{a}}$ & $\mathrm{X}$ \\
\hline Smoking status & $X^{a}$ & $\mathrm{X}$ \\
\hline Total protein & $\mathrm{X}^{\mathrm{b}}$ & \\
\hline UACR & & $\mathrm{X}$ \\
\hline
\end{tabular}

CYP cytochrome P450 3A4, eGFR estimated glomerular filtration rate, EPI CKD Epidemiology Collaboration equation, GGT gamma glutamyl transferase, GLP-1 glucagon-like peptide-1, $H b A 1 c$ hemoglobin A1c, $M D R D$ Modification of Diet Renal Disease study equation, $P F s$ prognostic factors, $P K$ pharmacokinetic, $S G L T 2$ sodium-glucose co-transporter-2, TTE time-to-event, UACR urine albumin-to-creatinine ratio

${ }^{\text {a }}$ Tested in the PK model

${ }^{b}$ Not tested in the PK model as the visual inspection of empirical Bayes estimate plots did not show a clear correlation. If not indicated otherwise, covariates refer to baseline values 


\subsection{Time-to-Event Model Development}

To characterize the effect of finerenone on the time to first occurrence of the composite kidney endpoint, a parametric TTE model was developed. The model development was performed in a stepwise approach using the interim dataset (Fig. 1) [20]. First, a placebo model was developed. Potential prognostic factors (PFs) for the baseline hazard, i.e., treatment-independent hazard (Table 1), were identified using the Cox proportional hazard analysis. All statistically significant PFs at a 0.05 level were included in a parametric hazard model after appropriate lumping (coined 'the full model'). The full model was run using different hazard shapes (detailed in the ESM) to select the optimal parametric hazard model. Subsequently, non-significant PFs were removed via backward deletion (significance at 0.01 level).

Upon inclusion of the active treatment data, the ER relationship was investigated using a maximum effect $\left(E_{\max }\right)$ model. Individual predicted finerenone concentration-time profiles based on the post-hoc estimates from the PK model considering the individual actual dosing history, the individual finerenone PK parameters of the subjects, and their covariates were used as input for the ER model. For subjects from the full analysis set without PK parameters, typical PK parameters were used considering their individual covariate values. As part of the ER investigation, it was tested whether inclusion of a delay and/or Hill coefficient in the $E_{\max }$ function would further improve the fit. After inclusion of the ER relationship, PFs that were removed from the placebo

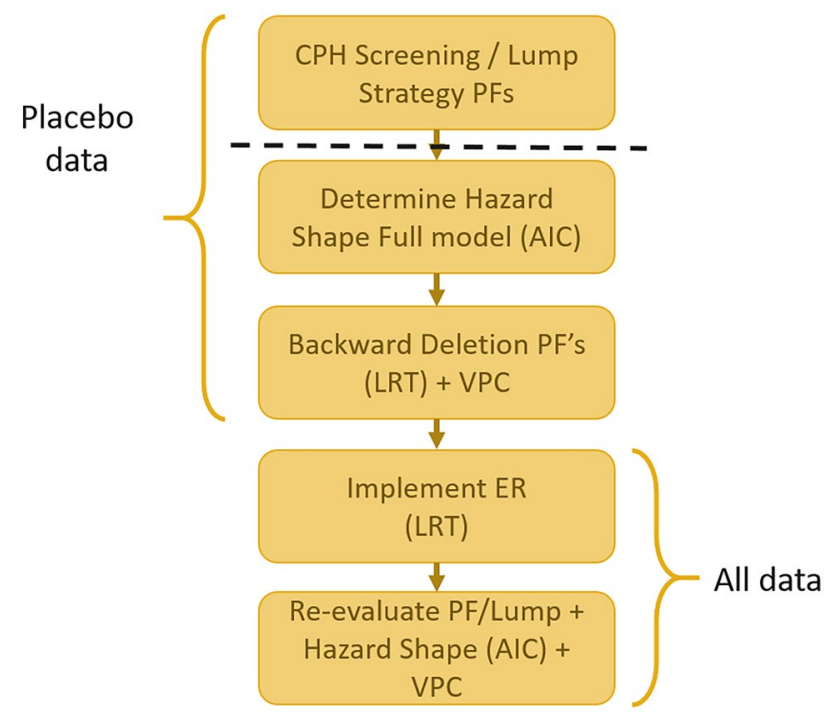

Fig. 1 Time-to-event model development workflow. Dashed black line indicates the switch from non-parametric to parametric models. $A I C$ Akaike information criterion, $C P H$ Cox proportional hazard, $E R$ exposure-response, $L R T$ likelihood ratio test, $P F$ prognostic factor, $V P C$ visual predictive check model during backward deletion were re-investigated and re-included if significant at a 0.01 level.

Upon availability of the final data, parameters were reestimated. Further details are provided in the ESM.

\subsection{Model Evaluation}

The performance of the PK model was evaluated by comparing simulations with the observed data, from here on referred to as the prediction-corrected visual predictive check [21]. The prediction-corrected visual predictive check simulations include residual error and inter-individual variability, but not parameter uncertainty.

Predictive performance of the TTE model was evaluated by means of visual predictive checks using an observed dropout. Using the parameter estimates of the final model, the individual survival probability curves were calculated for each subject in the full analysis set, taking individual PK parameters, dosing history, and prognostic factors into account. Censoring of simulated TTE was based on the individual observed censoring times, if applicable, or the end of the study if the subject was not censored in the original dataset. Uncertainty of the parameter estimates was not considered.

\section{Results}

\subsection{Clinical Study}

Out of 5734 patients randomized, 60 were prospectively excluded from all analyses because of critical Good Clinical Practice violations. Of the 5674 subjects valid for analysis in the FIDELIO-DKD study population, 2841 were randomized to placebo and 2833 to finerenone (2622 of whom started on a $10 \mathrm{mg}$ dose and $211 \mathrm{on}$ a $20 \mathrm{mg}$ dose). The median (5th-95th percentiles) eGFR and urine albumin-tocreatinine ratio (UACR) at baseline in FIDELIO-DKD were $43.0(26.7-66.9) \mathrm{mL} / \mathrm{min} / 1.73 \mathrm{~m}^{2}$ and $852(140-3366) \mathrm{mg} / \mathrm{g}$, respectively. More details on baseline characteristics have been published [2]. The average dose level over time was $15.1 \mathrm{mg}$ [3]. Based on post-baseline serum potassium observations, $34.8 \%$ of these were at the $10 \mathrm{mg}$ dose level, $48.7 \%$ at the $20 \mathrm{mg}$ dose level, and $16.5 \%$ during (permanent or temporary) treatment interruption [19].

\subsection{Population PK Modeling and Simulation}

The interim dataset used for the analysis included a total of 4308 valid finerenone concentrations from 2250 subjects. The final PK dataset used for the analysis contained 5057 valid finerenone concentrations from 2284 subjects. After a stepwise model development on the interim dataset, a 
two-compartmental PK model with volumes set equal (see Methods in the ESM) and absorption through a series of transit compartments and first-order elimination [17] adequately captured the finerenone concentration-time data in FIDELIO-DKD (Fig. 2).

Compared to the ARTS-DN model [17] that was used as the starting point for model development, the structural model was unchanged and the inter-individual variability parameter on the absorption rate was dropped as data were not sufficiently informative. Significant covariates included in the ARTS-DN model were bodyweight on the volume of distribution (V/F) and eGFR derived using the Modification of Diet Renal Disease study equation (eGFR-MDRD) [22-24] on apparent clearance (CL/F) and bioavailability (F). Replacing eGFR-MDRD by eGFR derived according to the CKD Epidemiology Collaboration (eGFR-EPI) [25, 26] did not result in a significant change; however, an exchange by time-varying eGFR-EPI improved the model fit. Hence, the effect of eGFR on CL/F and F was retained in the model as was the effect of bodyweight on V/F. This model was used as a starting point for a further covariate analysis (see also the ESM).

In addition to bodyweight, race/ethnicity (Korean subjects only) was found to have a statistically significant influence on V/F, while in addition to time-varying eGFR, body height, serum creatinine, smoking status, cytochrome P450 (CYP) 3A4 inhibitor use, and sodium-glucose transport protein 2 inhibitor (SGLT2i) use showed a statistically significant effect when applied on both CL/F and F (which was superior to an effect on CL/F only and motivated by the high first-pass clearance of finerenone [11]). Furthermore, gamma glutamyl transferase was found to have a statistically significant influence on CL/F. A summary plot illustrating the influence of the identified covariate effects on Maximum finerenone plasma concentration at steady-state after once daily dosing $\left(\mathrm{C}_{\max , \mathrm{md}}\right)$ and Area under the finerenone plasmaconcentration-time curve within a $24 \mathrm{~h}$ dosing interval at steady-state after once daily dosing $\left(\mathrm{AUC}_{\tau, \mathrm{md}}\right)$ is shown in Fig. 3. For continuous covariates, $\mathrm{C}_{\max , \mathrm{md}}$ and $\mathrm{AUC}_{\tau, \mathrm{md}}$ at the 5th-95th percentiles of the covariate distribution were within the general acceptance range for equivalence of $80-125 \%$ of the exposure at the median covariate value. For the categorical covariates, the largest effects were found for Korean subjects on $\mathrm{C}_{\text {max,md }}(21.2 \%$ decrease, no impact on $\mathrm{AUC}_{\tau, \mathrm{md}}$ ) and long-term (i.e., more than $50 \%$ of the time taking finerenone) SGLT2i users on $\mathrm{AUC}_{\tau, \mathrm{md}}(17.1 \%$ decrease, 9.73\% decrease in $\mathrm{C}_{\text {max,md }}$ ). Overall, all covariate effects are small and within or close to the typical equivalence range.

Observed and predicted median and variability of the concentration-time profile closely resemble each other (Fig. 2) supporting that the final population PK model described all data adequately. The pharmacokinetics were dose linear over the investigated dose range. Apart from the small influence of time-varying eGFR-EPI on CL/F and $\mathrm{F}$, the pharmacokinetics of finerenone were not found to be time dependent. The relevant half-life for steadystate exposure and accumulation $\left(\mathrm{t}_{1 / 2, \alpha}\right)$ was estimated at $2.7 \mathrm{~h}$, indicating near steady-state conditions are reached after the second dose of finerenone. Final model parameter estimates are shown in Table 2 and the final model code is provided in the ESM. The condition number was 183 , indicating that the model was stable and without collinearity in its parameters.

Simulations were performed to compare the exposure of finerenone at steady state in subgroups of interest (stratified for the following covariates: gender, age,
Fig. 2 Prediction-corrected visual predictive check of the pharmacokinetic model for finerenone. Black/gray dots: prediction-corrected observations; red line: observed median; red dashed lines: 2.5 th (as long as above the lower limit of quantification) and 97.5th percentiles of observations; red area: $95 \%$ variabilitybased prediction interval of the simulated medians; gray area: 95\% prediction interval; blue symbols: observations below the lower limit of quantification. CI confidence interval

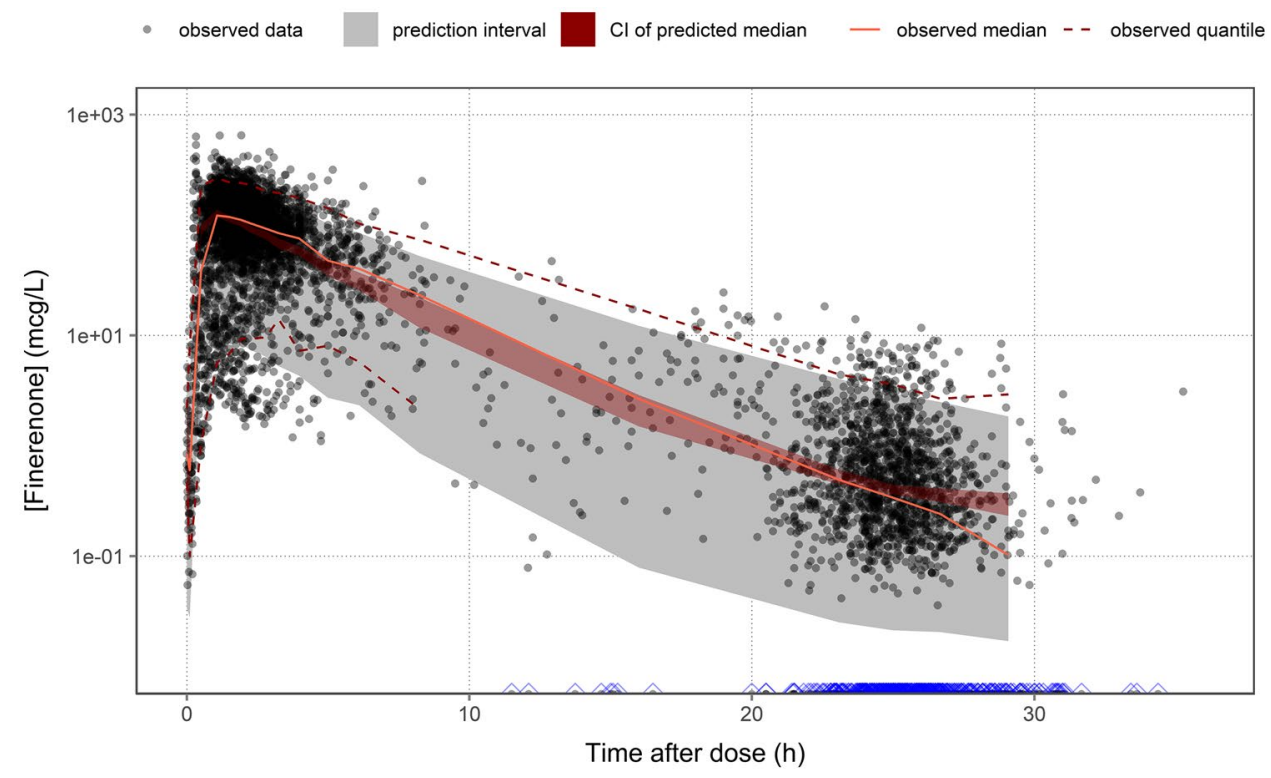




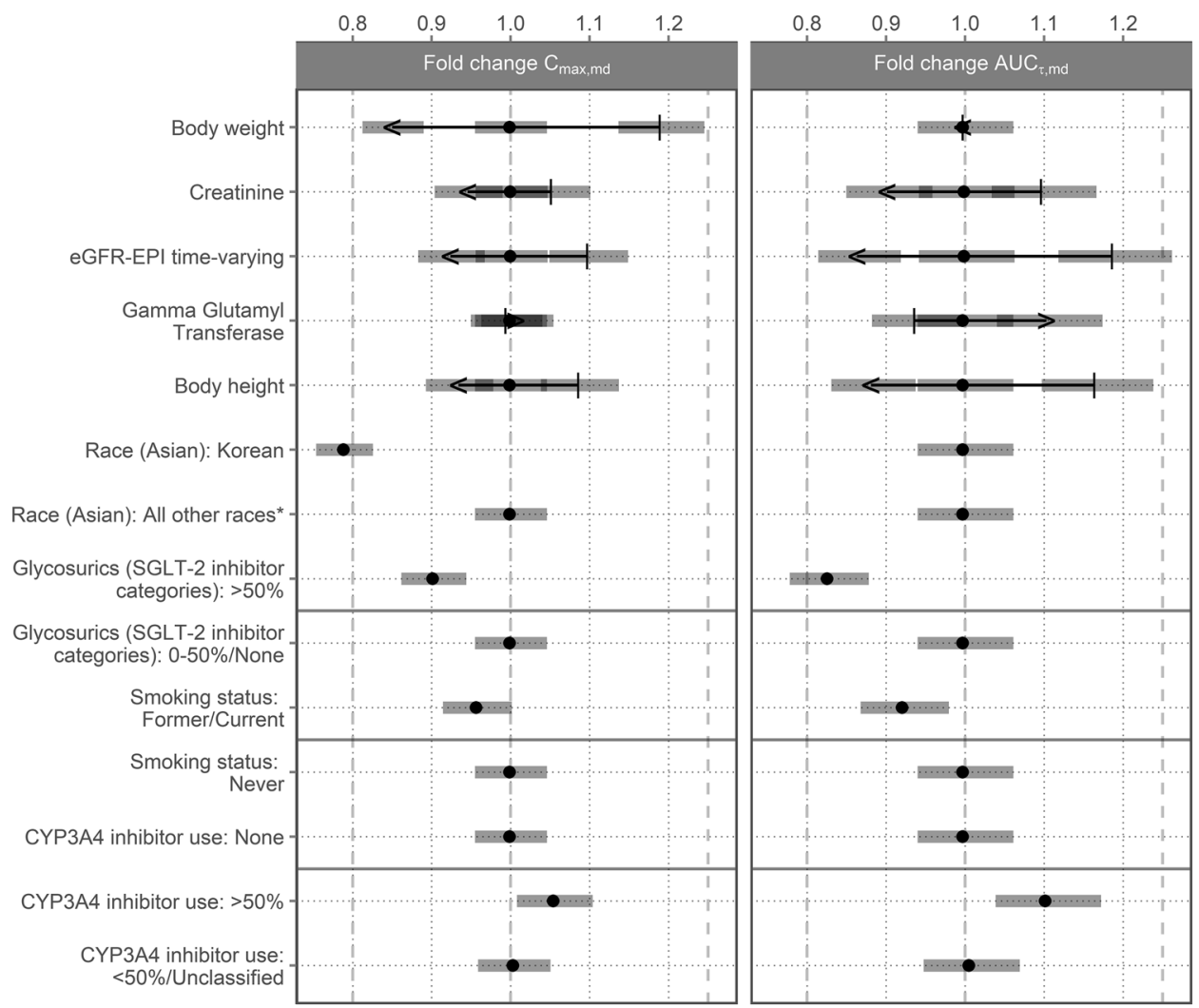

Fig. 3 Forest plots illustrating the influence of the identified covariate effects on $\mathrm{C}_{\mathrm{max} \text {,md }}$ and $\mathrm{AUC}_{\tau, \mathrm{md}}$ relative to the median covariate value (continuous covariates) or reference subgroup (categorical covariates). Black dots indicate the reference or the fold change relative to the reference. Whiskers, shaped as an arrow, indicate the pharmacokinetic parameter values at the 5th-95th percentiles of the covariate distribution, where the rear end of the arrow corresponds to the 5th percentile and the tip of the arrow corresponds to the 95th percentile of the covariate distribution. Gray bars indicate the uncertainty (5th-95th percentiles of the simulated 5th percentile, median, and

country, race, ethnicity, renal impairment categories). Figure 4 shows that ratios of the median exposure $\left(\mathrm{C}_{\mathrm{max}, \mathrm{md}}\right.$ and $\mathrm{AUC}_{\tau, \mathrm{md}}$ ) for a specific racial or ethnic group, including Koreans, and the reference (subject with median exposure) is always contained within the $80-125 \%$ range for both the post-hoc-based exposure as well as the exposure based on 1000 trial simulations. This was also the case for all the other subgroups of interest (provided as Figs. S2-S5 of the ESM).

\subsection{TTE Model Analysis}

The interim and final datasets used for the analysis contained a total of 5659 and 5674 subjects, respectively. During a median follow-up of 2.6 years, a kidney event contributing to the composite endpoint occurred in 504 of 2833 patients 95th percentile based on 10,000 simulated pharmacokinetic curves). Vertical dashed lines indicate unity and general acceptance range for equivalence of $0.8-1.25 . A U C_{\tau, \text { md }}$ Area under the finerenone plasmaconcentration-time curve within a $24 \mathrm{~h}$ dosing interval at steady-state after once daily dosing, $C_{\text {max,md }}$ Maximum finerenone plasma concentration at steady-state after once daily dosing, CYP cytochrome $\mathrm{P} 450$, $e G F R-E P I$ estimated glomerular filtration rate derived according to the CKD Epidemiology Collaboration, SGLT-2 sodium-glucose cotransporter-2

(17.8\%) in the finerenone group and in 600 of 2841 patients $(21.1 \%)$ in the placebo group.

The kidney composite TTE data were best described with a Weibull hazard model. The ER relationship was implemented as an $\mathrm{E}_{\max }$ model with finerenone concentration in the central compartment driving the response with neither a Hill coefficient nor a delay. Final model parameter estimates are shown in Table 3 and the final model code is provided in the ESM. In general, the final kidney TTE model captured the ER adequately (Fig. 5 and Figs. S8-S15 of the ESM). The condition number was 1078, which indicates the presence of some collinearity in the parameter estimates. This likely reflects the correlation of the two Weibull function parameters and was accepted because all other statistical criteria for model acceptance were met. 
Table 2 Parameter estimates and uncertainties of the finerenone pharmacokinetic model

\begin{tabular}{|c|c|c|}
\hline Parameter name & Estimate & $\operatorname{RSE}(\%)$ \\
\hline $\mathrm{K}_{\mathrm{a}}(1 / \mathrm{h})$ & 22.5 & 16.2 \\
\hline $\mathrm{CL} / \mathrm{F}(\mathrm{L} / \mathrm{h})$ & 29.9 & 3.62 \\
\hline $\mathrm{V}_{\mathrm{c}} / \mathrm{F}(\mathrm{L})$ & 113 & 2.79 \\
\hline $\mathrm{Q} / \mathrm{F}(\mathrm{L} / \mathrm{h})$ & 0.335 & 9.28 \\
\hline Ratio $\mathrm{V}_{\mathrm{p}} / \mathrm{F}$ and $\mathrm{V}_{\mathrm{c}} / \mathrm{F}$ & 1 (fixed) & - \\
\hline Absorption lag time (h) & 0.215 (fixed) & - \\
\hline Relative bioavailaility & 1 (fixed) & - \\
\hline Effect of body weight on $V_{c} / F$ & 0.501 & 9.87 \\
\hline Effect of eGFR-EPI (time-varying) on CL/F and F & 0.155 & 20.1 \\
\hline Effect of body height on CL/F and F & 0.720 & 16.1 \\
\hline Effect of creatinine on $\mathrm{CL} / \mathrm{F}$ and $\mathrm{F}$ & 0.118 & 38.4 \\
\hline Effect for Korean subjects on $V_{c} / F$ & 1.29 & 6.79 \\
\hline Effect of SGLT2 inhibitor use ( $\geq 50 \%$ of on treatment period) on $\mathrm{CL} / \mathrm{F}$ and $\mathrm{F}$ & 1.10 & 2.23 \\
\hline Effect of smoking (current or former smokers) on CL/F and $\mathrm{F}$ & 1.04 & 1.11 \\
\hline Effect of GGT on CL/F & -0.0694 & 16.2 \\
\hline $\begin{array}{l}\text { Effect of CYP3A4 inhibitor use (weak, moderate, or strong } \geq 50 \% \text { of on-treat- } \\
\text { ment period) on CL/F and } F\end{array}$ & 0.951 & 1.66 \\
\hline Effect of CYP3A4 inhibitor use (other categories) on CL/F and F & 0.996 & 2.17 \\
\hline \multicolumn{3}{|l|}{ Inter-individual variability } \\
\hline$\omega^{2} \mathrm{CL} / \mathrm{F}^{\mathrm{a}}$ & 0.0961 & 7.30 \\
\hline$\omega^{2} \mathrm{~V}_{\mathrm{c}} / \mathrm{F}^{\mathrm{b}}$ & 0.104 & 18.4 \\
\hline Covariance $\mathrm{CL} / \mathrm{F} \times \mathrm{Vc} / \mathrm{F}$ & 0.0442 & 19.4 \\
\hline \multicolumn{3}{|l|}{ Residual error } \\
\hline$\sigma^{2}$ & 0.313 & 2.83 \\
\hline
\end{tabular}

$C L$ clearance, $C V$ coefficient of variation, $C Y P$ cytochrome $\mathrm{P} 450, e G F R-E P I$ estimated glomerular filtration rate according to the CKD Epidemiology Collaboration equation, $F$ bioavailability, $G G T$ gamma glutamyl transferase, $K_{a}$ absorption rate constant, $Q$ intercompartmental clearance, $R S E$ relative standard error of estimate, $S G L T 2$ sodium-glucose co-transporter-2, $V_{c}$ central volume of distribution, $V_{p}$ peripheral volume of distribution

${ }^{\text {a }}$ Shrinkage was $26.6 \%$, variability expressed as CV: $31.8 \%$

${ }^{\mathrm{b}}$ Shrinkage was $47.8 \%$, variability expressed as CV: $33.0 \%$
The UACR was the most significant PF influencing the baseline hazard (that applies to both treatment groups), with a $85.0 \%$ decrease and a $324 \%$ increase of the hazard relative to median at the 5 th and 95 th percentiles of the UACR distribution, respectively. Additional PFs were: eGFR-EPI at baseline ( $+30.2 \%$ to $-21.7 \%$ at 5 th-95th percentiles), body mass index $(+22.8 \%$ to $-22.2 \%$ at 5 th-95th percentiles), age ( $+36.9 \%$ to $-18.4 \%$ at 5 th-95th percentiles), race/ethnicity (African American $+102 \%$, all Asian except Japanese $+20.3 \%$, compared to all other races/ethnicities including Japanese), long-term use of SGLT2i treatment ( $-50.7 \%$ vs no use), any GLP-1 agonist use ( $-34.2 \%$ vs no use), and hepatic impairment (+69.9\% for individuals likely Child Pugh B vs likely Child Pugh A or no impairment). The magnitude of these PFs is illustrated in Figs. S6 and $\mathrm{S} 7$ of the ESM.
The visual predictive checks of the final kidney TTE model, stratified by exposure (defined as average exposure from randomization until event) quartiles, are shown in Fig. 5. In general, the observed Kaplan-Meier for each exposure quartile was contained within the $95 \%$ prediction interval. The event rate slightly decreased with higher finerenone exposure. A slight overprediction of the event rate in the first 2 years in the placebo group is observed. In addition, visual predictive checks stratified by PFs in general indicated that the TTE data are adequately captured across the PF ranges (continuous PFs) and categories (categorical PFs) [Figs. S8-15 of the ESM].

In general, the final kidney TTE model captured the ER relationship adequately. The half-maximal drug effect was estimated at $0.166 \mu \mathrm{g} / \mathrm{L}$ and the maximum decrease of the hazard was estimated at $36.1 \%\left(\mathrm{E}_{\max }\right)$. 
Fig. 4 Comparison of $\mathrm{C}_{\text {max,md }}$ and $\mathrm{AUC}_{\tau, \mathrm{md}}$ between racial and ethnic groups relative to the overall population. The $\%$ indicates the percentage of subjects within each group when compared to the total population of subjects in analyzed data. Red dots and whiskers represent the median and 5th-95th percentiles of the $\mathrm{C}_{\text {max,md }}$ and $\mathrm{AUC}_{\tau \text {,md }}$ ratio using a subject with median $\mathrm{C}_{\text {max,md }}$ and $\mathrm{AUC}_{\tau, \mathrm{md}}$ as reference, based on 1000 trial simulations. The red areas indicate the uncertainty (5th-95th percentiles of the simulated 5 th percentile, median, and 95th percentile). Blue dots and whiskers represent the median and 5th-95th percentiles of the $\mathrm{C}_{\text {max,md }}$ and $\mathrm{AUC}_{\tau, \mathrm{md}}$ ratio using a subject with median $C_{\max , m d}$ and $\mathrm{AUC}_{\tau, \mathrm{md}}$ as reference, based on the posthoc estimates. Vertical dashed lines indicate unity and the $0.8-1.25$ lines

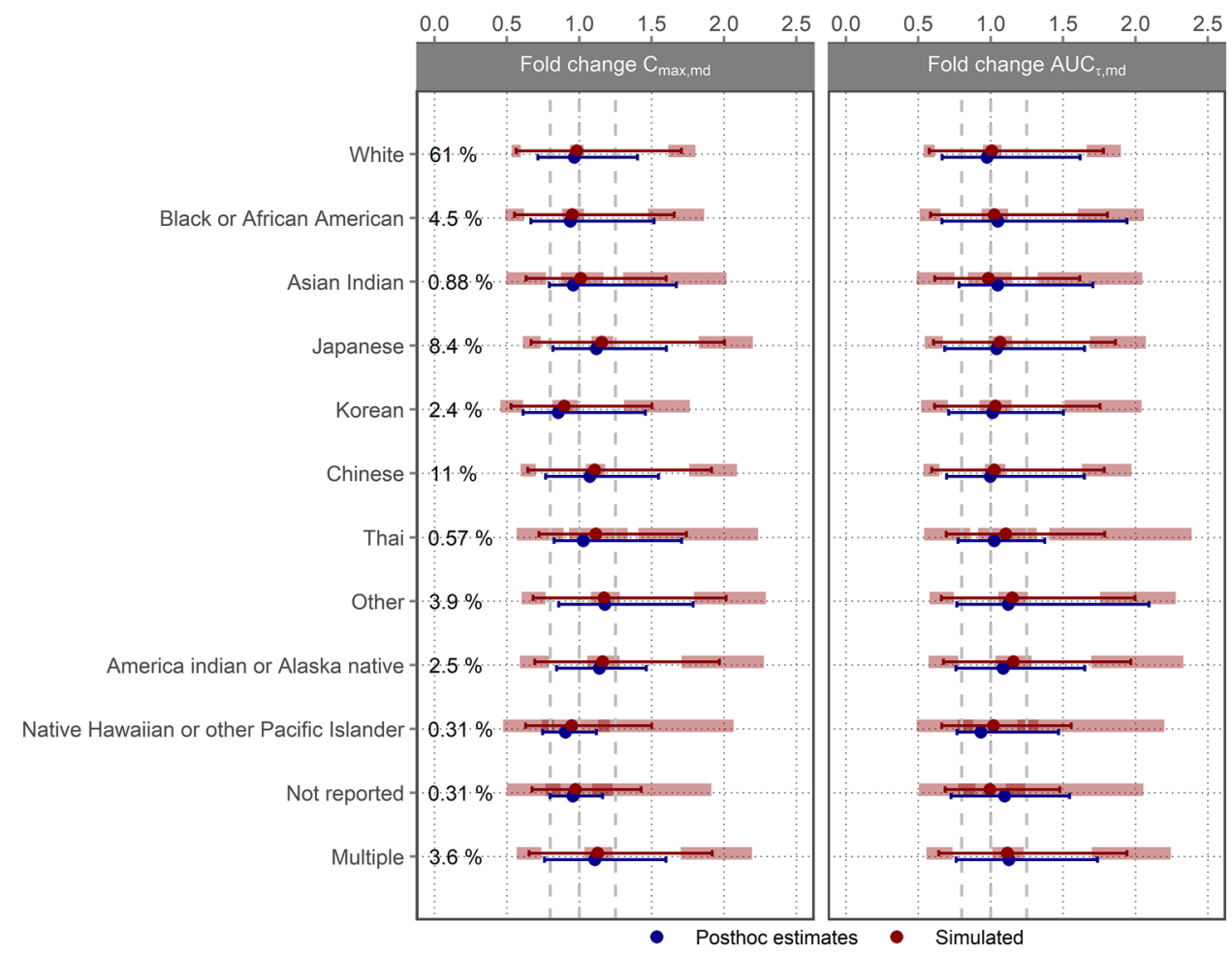

Table 3 Parameter estimates and uncertainties of the time-to-event model for the renal composite endpoint

\begin{tabular}{lll}
\hline Parameter name & Estimate & RSE (\%) \\
\hline Lambda (log scale, Weibull hazard) & -19.2 & 2.50 \\
Alpha (Weibull hazard) & 1.80 & 2.76 \\
$\mathrm{E}_{\text {max }}$ & -0.361 & 12.9 \\
EC $_{50}$ & 0.166 & 16.9 \\
HILL & 1 (fixed) & - \\
Age (log-transformed) & -1.13 & 17.8 \\
Body mass index (log-transformed) & -0.747 & 23.4 \\
eGFR-EPI (log-transformed) & -0.554 & 20.7 \\
UACR (log-transformed) & 1.05 & 4.50 \\
Likely/certain Child-Pugh B & 0.699 & 23.8 \\
SGLT2 inhibitor use $>50 \%$ of on treatment & -0.507 & 21.1 \\
period & & \\
Any on-treatment GLP-1 agonist use & -0.342 & 21.3 \\
Black/African-American & 1.02 & 24.4 \\
All Asian ethnicities, except Japanese & 0.203 & 45.0 \\
\hline
\end{tabular}

$E C_{50}$ half-maximal drug effect, $e G F R-E P I$ estimated glomerular filtration rate derived according to the CKD Epidemiology Collaboration, $G L P-1$ glucagon-like peptide-1, RSE relative standard error of estimate, $S G L T 2$ sodium-glucose co-transporter-2, UACR urine albumin-to-creatinine ratio

\section{Discussion}

\subsection{Pharmacokinetics}

The population PK analysis of the pivotal phase III study supports the finding from phase IIb analysis that, overall, the pharmacokinetics are similar in patients of the target population with CKD and T2D (ARTS-DN and FIDELIO-DKD), phase IIa patients (ARTS) and healthy participants in phase I studies, if differences in covariates, especially eGFR, are taken into account [17].

While in previous studies only selected covariates were investigated, a more comprehensive covariate screen was conducted based on the phase III data. The identified covariates are visualized in Fig. 3. Bodyweight and eGFR were already identified based on phase IIb data [17]. In the current analysis, eGFR calculated based on the 'CKD Epidemiology Collaboration' (CKD-EPI) formula [25, 26] performed comparably to eGFR calculated based on the 'Modification of Diet in Renal Disease' (CKD-MDRD) formula used historically [22-24]. However, time-varying eGFR (based on CKD-EPI) was superior to baseline eGFR for reasons further discussed below. Interestingly, also serum creatinine was 


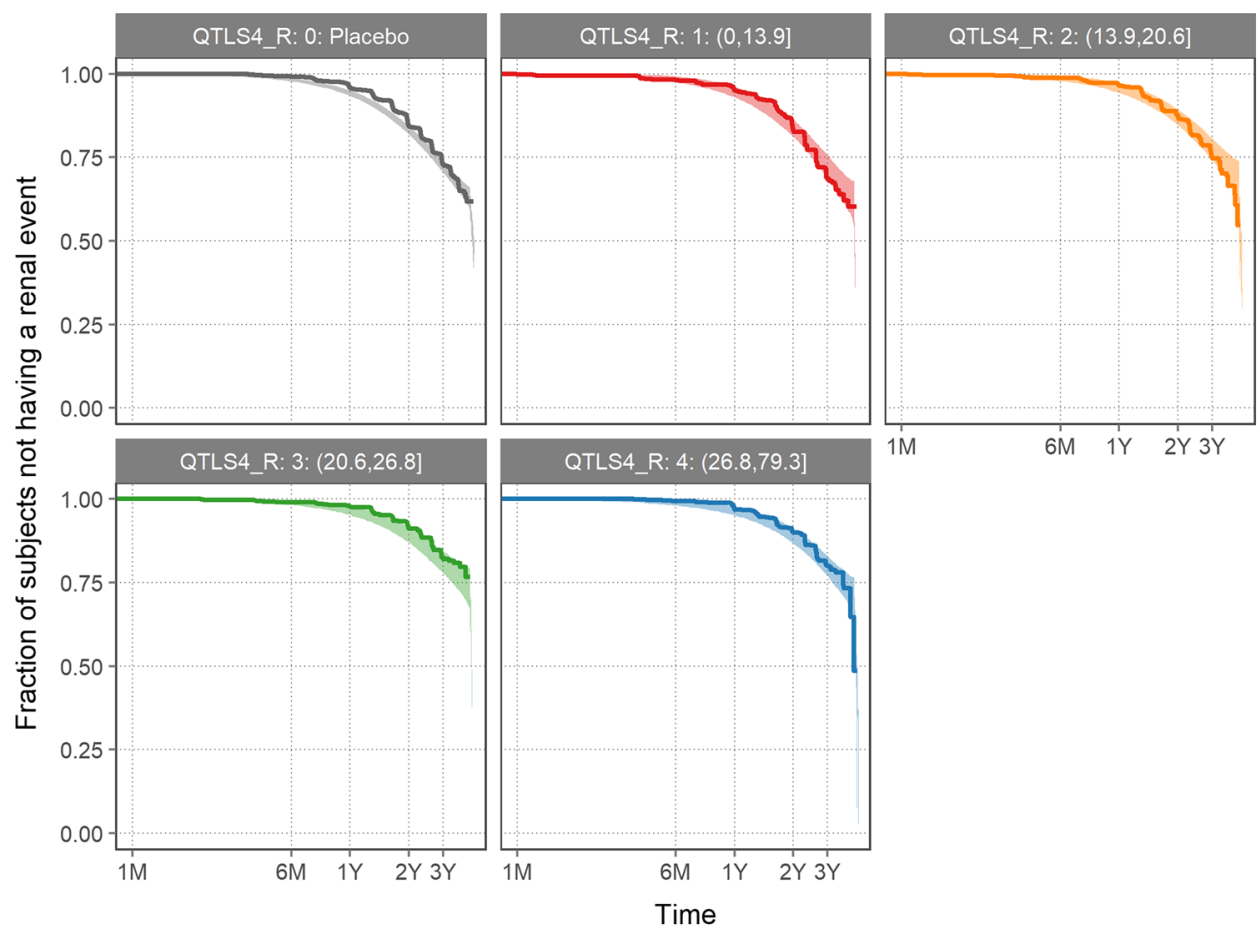

Fig. 5 Visual predictive check plot of the kidney time-to-event model applied to FIDELIO-DKD data. The study was replicated 1000 times, where individual time-to-event sampling was based on a numerical inversion of the individual survival probability curve using a granularity of 7 days. This resulted in 1000 Kaplan-Meier (KM) curves consisting of 5674 subjects each. The 2.5 th- 97.5 th percentiles of these KM curves were calculated, plotted, and overlaid with the observed KM curve. Next to the fit of the placebo data (gray), the

identified as an independent covariate on clearance next to eGFR, compensating for parts of the eGFR effect. Because of the negligible renal clearance of finerenone, kidney markers such as eGFR and creatinine should be considered as surrogates representing a more complex underlying pathophysiology where likely accumulating uremic toxins ultimately impair CYP3A4 metabolism [27]. The small effect of SGLT2i use on clearance might also be attributable to this phenomenon, as at least pre-clinical data indicate that SGLT2i use may partly reverse this pathophysiology [28]. Use of CYP3A4 inhibitors showed a smaller effect than expected based on finerenone metabolism and phase I studies [11], which is not untypical for co-medication effects in phase III studies as, for example, co-medication dosing, timing, and PK sampling are naturally not optimized to show maximal effects. Additionally, the minor and clinically not relevant effect of gamma glutamyl transferase as a liver marker can possibly be attributed to an effect on hepatic metabolization in line with small effects observed in the hepatic impairment study [12]. The small influence of smoking status on clearance is difficult to explain considering the known metabolism pathways of finerenone (via finerenone-treated subjects were divided into four exposure quartiles based on average concentration until (censored) event. The QTLS4_R numbers at the top indicate the exposure ranges in $\mu \mathrm{g} / \mathrm{L}$. Thick lines indicate the observed KM curves, where, owing to the visit-related nature of the event, the left corner of each step should be used to evaluate the goodness of fit relative to the ribbon, which indicates the 95\% PI. PI Prediction Interval

CYP3A4 and CYP2C8, both not known for being induced by smoking) and may reflect a more complex health impact. In addition to bodyweight on volume, the current analysis identified body height as an additional size descriptor covariate, however, affecting clearance and bioavailability. While discussions on allometric rules and optimal body size descriptors to relate to clearance are ongoing [29,30], it should be noted that, generally, different size descriptors are correlated and identifying one over the other may have various reasons. The analysis of different races and ethnicities only showed an effect in Korean individuals on the volume that is not straightforward to explain. This may be a spurious finding based on limited data (only $2.4 \%$ of subjects were Korean).

While several covariates were statistically significant on the pre-specified level, either covariate effects themselves or forward simulations of their effects in special subgroups of interest, which considered the multivariate distribution of additional covariates, were contained within typical equivalence ranges (80-125\%) including Korean ethnicity (see Fig. 4). Generally, statistical significance may be achieved also based on the large number of patients in total; however, 
the small effect sizes indicate that no covariate was clinically significant in FIDELIO-DKD.

In line with previous analyses [14, 17], the current analysis supports that the pharmacokinetics of finerenone are dose linear. As the eGFR of patients declines over time, reflecting both the progressive nature of CKD and age-related decline in kidney function, this introduces a small time dependency in the pharmacokinetics of finerenone on long time scales as identified in the current analysis by time-varying eGFR as a covariate, which was not observed in previous studies with a shorter duration. Overall, the final population PK model adequately captured the typical pharmacokinetics of finerenone and its variability in FIDELIO-DKD and allowed for the estimation of individual PK parameter post-hoc estimates to support subsequent ER analyses.

\subsection{Exposure vs Kidney Outcome Events}

The ER analyses of the primary kidney endpoint confirmed the treatment benefit [3] and showed that patients with higher finerenone exposure had a small additional benefit from treatment. However, with a half-maximal drug effect estimated at $0.166 \mu \mathrm{g} / \mathrm{L}$, most patients receiving the higher finerenone dose exceed that level for the entire dosing interval. Therefore, the effects on the primary endpoint appear largely saturated (Fig. S16 of the ESM). The saturation of kidney benefits towards $20 \mathrm{mg}$ of finerenone once daily is qualitatively in line with the UACR response observed in the ER analysis of phase IIb data approaching saturation [17].

The maximal hazard decrease for the primary endpoint is estimated at $36.1 \%$, assuming continuous treatment on a maximally effective dose. While the primary statistical efficacy analysis indicated a risk reduction of $18 \%$, an on-treatment sensitivity analysis indicates a larger risk reduction of $22 \%$ [3]. Ontreatment analysis included events that occurred during phases where dosing was interrupted and excluded events that occurred more than 30 days after permanent drug discontinuation. In addition, not all subjects received the $20 \mathrm{mg}$ dose continuously. Analysis of the secondary endpoint, doubling of serum creatinine (corresponding to an sustained eGFR decrease $\geq 57 \%$ ), indicated a reduction of $32 \%$ under finerenone treatment [3], an endpoint that was part of the primary composite in other recent CKD studies such as CREDENCE [31]. These observations highlight the potential of finerenone to significantly improve the treatment of patients with CKD.

The parametric TTE model indicated a slight overprediction of the event rate of the primary kidney endpoint in the first 2 years in the placebo group, which may be explained by an initial eGFR decline after start of treatment not included in the model. An initial decline in eGFR is observed with initiation of MRAs as well as with renin-angiotensin system inhibitors and SGLT2is. This decline is postulated to reflect reduced intraglomerular pressure, and correlates with long-term preservation of kidney function $[32,33]$. The hypothesis that this may impact the time to a primary kidney event is supported by explorations indicating an improved fit of the placebo data when considering the initial eGFR decline (data not shown). However, overall effects are small, also with respect to conclusions. With more detailed eGFR analysis including data from the recently published FIGARO-DKD study [9], this aspect may be further evaluated and the models independently validated.

Eight PFs were identified for the treatment independent hazard, i.e., also independent of finerenone exposure. High UACR is an established independent prognostic factor for kidney events and a marker of kidney damage and inflammation, and was the most significant PF. Similarly, low eGFR (at baseline) is an established independent risk factor for further progression towards kidney failure and was a significant $\mathrm{PF}$ as well. Body mass index and age have a counter-intuitive effect with a higher risk for kidney events associated with lower age and lower body mass index, which is difficult to explain. However, the magnitudes of these effects were relatively small. African American ethnicity is a PF that has been observed previously and may be related to a high burden of co-morbidities and access to healthcare [34, 35]. Moderate hepatic impairment also is a PF, likely reflecting the impact of the co-morbidity on the risk for these patients per se, while not significantly influencing the pharmacokinetics in FIDELIO-DKD.

During the conduct of FIDELIO-DKD, evidence of cardiorenal protection in patients with CKD was also observed with SGLT2is in other phase III trials $[31,36]$. As shown in the FIDELIO-DKD analysis here, SGLT2i use reduced the risk of a kidney event independent of finerenone treatment by $50.7 \%$ (95\% confidence interval 29.8-71.6). This effect size is even larger than what has been reported from, for example, the CREDENCE trial where MRA use was excluded and may reflect confounding factors not reflected in the multivariate analysis [31]. As the primary statistical subgroup analysis was not conclusive on the benefits of finerenone in the subgroup of patients receiving SGLT2is at baseline [3], SGLT2i use was additionally (post-hoc) tested for its potential to modify the treatment effect of finerenone. No significant effect of SGLT2i use on the effect of finerenone on the primary endpoint per se was found. These analyses therefore indicate independent and additive effects of finerenone and SGLT2is on the primary kidney endpoint. The more conclusive findings of the ER analysis compared with the statistical sub-group analysis can be explained by differences in the SGLT2i use definition (in the subgroup analysis, baseline use only was considered, while in this analysis, long-term treatment use was considered), the difference between TTE approaches (non-parametric in the subgroup analysis compared to the parametric approach allowing for inclusion of several PFs and thus multivariate analysis), and finally, the higher power of identifying an effect when considering treatment as a continuous exposure rather than a binary variable only. The finding 
could be important for patients and prescribers as it supports a potential use of a SGLT2 $i$ on top of finerenone, or vice versa. As a potential limitation it should be noted that the number of SGLT2 $i$ users as defined here was limited as well, and longterm treatment use did not consider exact drug, time, duration, or dose regimen information of concomitant SGLT2i use. Thus, refined analysis and future data could be used to further substantiate this finding.

\section{Conclusions}

In summary, the analyses support the previous analysis indicating that the pharmacokinetics of finerenone is overall well characterized. In FIDELIO-DKD, no clinically relevant covariates for pharmacokinetics were identified. The exposure vs TTE analyses are in line with the primary efficacy analysis, support the tested dosing regimen, and indicate an independent and additive benefit of finerenone and SGLT2i use.

Supplementary Information The online version contains supplementary material available at https://doi.org/10.1007/s40262-021-01082-2.

Acknowledgements The authors thank the FIDELIO-DKD Committees, investigators, and patients; Sissy Stauffenberg (ClinStat), Martin Gebel, Cosima Klein, Aziz Tuermer, Jacobus Buytendach, and Patrick Schloemer (Bayer) for derived variables, general information about data, and alignment with primary statistical analysis; Diana Hijdra (LAP\&P), Udo Kuhnert, and Nicholas Downie (Bayer) for supporting pharmacometric analysis dataset creation; Sven Hoefman and Peter Vis (LAP\&P) for contributions to the general TTE methodology; Richard Hooijmaijers (LAP\&P) for support with PK simulation scripts; Nurahamid Siddiki, Md. Aminul Islam, Rumana Khatun (Shafi Consultancy), and Anne Keunecke (LAP\&P) for quality control; Hauke Ruehs, Yang Zhang, and Alexander Solms (Bayer) for critical discussion of the methods and results; Meike Brinker and Susanne Metzger (Bayer) for critical discussion of the results; and Simone Steinbach and Ui Yen Morgenthaler (Bayer) for medical writing support.

\section{Declarations}

Funding LAP\&P conducted the analysis as contract research for Bayer. However, LAP\&P (PB, MR, EM, NS) was not paid to contribute to manuscript preparation.

Conflict of interest PB, MR, EM, are NS are employed by LAP\&P, a CRO working for Bayer. AS, RH, AJ, DG, JL, and TE are Bayer employees and potential shareholders of Bayer AG.

Ethics approval The study protocol was approved by international review boards, independent ethics committees, and competent authorities according to national and international regulations. FIDELIO-DKD was conducted in accordance with the ICH Harmonized Tripartite Guideline for Good Clinical Practice.

Consent to participate All study participants provided written informed consent before entering the study.
Consent for publication All study participants provided written informed consent before entering the study.

Availability of data and material Data will be made available in an electronic repository in future, at a date to be specified by Bayer.

Code availability The NONMEM analysis code is provided as ESM.

Author contributions PB: wrote manuscript, designed research, performed popPK/TTE analysis, analyzed data; MR: wrote manuscript, performed TTE analysis, analyzed data; EM: wrote manuscript, performed popPK, analyzed data; NS: wrote manuscript, designed research, supported popPK/TTE analysis, analyzed data; AS: wrote manuscript, designed research, programmed and analyzed data; $\mathrm{RH}$ : wrote manuscript, designed research; AJ: wrote manuscript, designed research; DG: wrote manuscript, designed research; JL: wrote manuscript, designed research; TE: wrote manuscript, designed research, steered analysis, analyzed data.

Open Access This article is licensed under a Creative Commons Attribution-NonCommercial 4.0 International License, which permits any non-commercial use, sharing, adaptation, distribution and reproduction in any medium or format, as long as you give appropriate credit to the original author(s) and the source, provide a link to the Creative Commons licence, and indicate if changes were made. The images or other third party material in this article are included in the article's Creative Commons licence, unless indicated otherwise in a credit line to the material. If material is not included in the article's Creative Commons licence and your intended use is not permitted by statutory regulation or exceeds the permitted use, you will need to obtain permission directly from the copyright holder. To view a copy of this licence, visit http://creativecommons.org/licenses/by-nc/4.0/.

\section{References}

1. Agarwal R, Anker SD, Bakris G, Filippatos G, Pitt B, Rossing P, et al. Investigating new treatment opportunities for patients with chronic kidney disease in type 2 diabetes: the role of finerenone. Nephrol Dial Transplant. 2020.

2. Bakris GL, Agarwal R, Anker SD, Pitt B, Ruilope LM, Nowack $\mathrm{C}$, et al. Design and baseline characteristics of the finerenone in reducing kidney failure and disease progression in diabetic kidney disease trial. Am J Nephrol. 2019;50(5):333-44.

3. Bakris GL, Agarwal R, Anker SD, Pitt B, Ruilope LM, Rossing $\mathrm{P}$, et al. Effect of finerenone on chronic kidney disease outcomes in type 2 diabetes. N Engl J Med. 2020;383(23):2219-29.

4. Filippatos G, Anker SD, Agarwal R, Pitt B, Ruilope LM, Rossing $\mathrm{P}$, et al. Finerenone and cardiovascular outcomes in patients with chronic kidney disease and type 2 diabetes. Circulation. 2020.

5. Allison SJ. Finerenone in chronic kidney disease. Nature Rev Nephrol. 2021;17(1):13.

6. Ingelfinger JR, Rosen CJ. Finerenone: halting relative hyperaldosteronism in chronic kidney disease. N Engl J Med. 2020;383(23):2285-6.

7. Grune J, Beyhoff N, Smeir E, Chudek R, Blumrich A, Ban Z, et al. Selective mineralocorticoid receptor cofactor modulation as molecular basis for finerenone's antifibrotic activity. Hypertension. 2018;71(4):599-608.

8. Lavall D, Jacobs N, Mahfoud F, Kolkhof P, Bohm M, Laufs U. The non-steroidal mineralocorticoid receptor antagonist finerenone prevents cardiac fibrotic remodeling. Biochem Pharmacol. 2019; 168:173-83. 
9. Pitt B, Filippatos G, Agarwal R, Anker SD, Bakris GL, Rossing $\mathrm{P}$, et al. Cardiovascular events with finerenone in kidney disease and type 2 diabetes. N Engl J Med. 2021.

10. Gerisch M, Heinig R, Engelen A, Lang D, Kolkhof P, Radtke $\mathrm{M}$, et al. Biotransformation of finerenone, a novel nonsteroidal mineralocorticoid receptor antagonist, in dogs, rats, and humans, in vivo and in vitro. Drug Metab Dispos. 2018;46(11):1546-55.

11. Heinig R, Gerisch M, Engelen A, Nagelschmitz J, Loewen S. Pharmacokinetics of the novel, selective, non-steroidal mineralocorticoid receptor antagonist finerenone in healthy volunteers: results from an absolute bioavailability study and drug-drug interaction studies in vitro and in vivo. Eur J Drug Metab Pharmacokinet. 2018;43(6):715-27.

12. Heinig R, Lambelet M, Nagelschmitz J, Alatrach A, Halabi A. Pharmacokinetics of the novel nonsteroidal mineralocorticoid receptor antagonist finerenone (BAY 94-8862) in individuals with mild or moderate hepatic impairment. Eur J Drug Metab Pharmacokinet. 2019;44(5):619-28.

13. Heinig R, Gerisch M, Bairlein M, Nagelschmitz J, Loewen S. Results from drug-drug interaction studies in vitro and in vivo investigating the effect of finerenone on the pharmacokinetics of comedications. Eur J Drug Metab Pharmacokinet. 2020;45(4):433-44.

14. Lentini S, Heinig R, Kimmeskamp-Kirschbaum N, Wensing G. Pharmacokinetics, safety and tolerability of the novel, selective mineralocorticoid receptor antagonist finerenone: results from first-in-man and relative bioavailability studies. Fundam Clin Pharmacol. 2016;30(2):172-84.

15. Heinig R, Kimmeskamp-Kirschbaum N, Halabi A, Lentini S. Pharmacokinetics of the novel nonsteroidal mineralocorticoid receptor antagonist finerenone (BAY 94-8862) in individuals with renal impairment. Clin Pharmacol Drug Dev. 2016;5(6):488-501.

16. Bakris GL, Agarwal R, Chan JC, Cooper ME, Gansevoort RT, Haller $\mathrm{H}$, et al. Effect of finerenone on albuminuria in patients with diabetic nephropathy: a randomized clinical trial. JAMA. 2015;314(9):884-94.

17. Snelder N, Heinig R, Drenth HJ, Joseph A, Kolkhof P, Lippert J, et al. Population pharmacokinetic and exposure-response analysis of finerenone: insights based on phase IIb data and simulations to support dose selection for pivotal trials in type 2 diabetes with chronic kidney disease. Clin Pharmacokinet. 2020;59(3):359-70.

18. Katayama S, Yamada D, Nakayama M, Yamada T, Myoishi M, Kato M, et al. A randomized controlled study of finerenone versus placebo in Japanese patients with type 2 diabetes mellitus and diabetic nephropathy. J Diabetes Complications. 2017;31(4):758-65.

19. Goulooze B, Snelder N, Seelmann A, Horvat-Broecker A, Brinker $\mathrm{M}$, Joseph A, et al. Finerenone dose-exposure-serum potassium response analysis of FIDELIO-DKD phase 3: the role of dosing, titration, and inclusion criteria. Clin Pharmacokinet. https://doi. org/10.1007/s40262-021-01083-1 (Accepted).

20. Grothey A, Hoefman S, Ruppert M, Vis P, Zisowsky J, FialaBuskies S, et al. Exploration of efficacious alternative regorafenib regimens to manage hand-foot-skin-reaction (HFSR). Ann Oncol. 2019;30(Suppl5):198-252.

21. Bergstrand M, Hooker AC, Wallin JE, Karlsson MO. Prediction-corrected visual predictive checks for diagnosing nonlinear mixed-effects models. AAPS J. 2011;13(2):143-51.
22. Levey AS, Coresh J, Greene T, Stevens LA, Zhang YL, Hendriksen $S$, et al. Using standardized serum creatinine values in the modification of diet in renal disease study equation for estimating glomerular filtration rate. Ann Intern Med. 2006;145(4):247-54.

23. Chudleigh RA, Ollerton RL, Dunseath G, Peter R, Harvey JN, Luzio S, et al. Performance of the revised " 175 " modification of diet in renal disease equation in patients with type 2 diabetes. Diabetologia. 2008;51(9):1714-8.

24. Matsuo S, Imai E, Horio M, Yasuda Y, Tomita K, Nitta K, et al. Revised equations for estimated GFR from serum creatinine in Japan. Am J Kidney Dis. 2009;53(6):982-92.

25. Horio M, Imai E, Yasuda Y, Watanabe T, Matsuo S. Modification of the CKD Epidemiology Collaboration (CKD-EPI) equation for Japanese: accuracy and use for population estimates. Am J Kidney Dis. 2010;56(1):32-8.

26. Levey AS, Stevens LA, Schmid CH, Zhang YL, Castro AF 3rd, Feldman HI, et al. A new equation to estimate glomerular filtration rate. Ann Intern Med. 2009;150(9):604-12.

27. Lalande L, Charpiat B, Leboucher G, Tod M. Consequences of renal failure on non-renal clearance of drugs. Clin Pharmacokinet. 2014;53(6):521-32.

28. Ali BH, Al Salam S, Al Suleimani Y, Al Za'abi M, Abdelrahman AM, Ashique M, et al. Effects of the SGLT-2 inhibitor canagliflozin on adenine-induced chronic kidney disease in rats. Cell Physiol Biochem. 2019;52(1):27-39.

29. Anderson BJ, Holford NH. What is the best size predictor for dose in the obese child? Paediatr Anaesth. 2017;27(12):1176-84.

30. Mansoor N, Ahmad T, Alam Khan R, Sharib SM, Mahmood I. Prediction of clearance and dose of midazolam in preterm and term neonates: a comparative study between allometric scaling and physiologically based pharmacokinetic modeling. Am J Ther. 2019;26(1):32-7.

31. Perkovic V, Jardine MJ, Neal B, Bompoint S, Heerspink HJL, Charytan DM, et al. Canagliflozin and renal outcomes in type 2 diabetes and nephropathy. N Engl J Med. 2019;380(24):2295-306.

32. Holtkamp FA, de Zeeuw D, Thomas MC, Cooper ME, de Graeff PA, Hillege HJ, et al. An acute fall in estimated glomerular filtration rate during treatment with losartan predicts a slower decrease in long-term renal function. Kidney Int. 2011;80(3):282-7.

33. Weir MR. Acute fall in glomerular filtration rate with renin-angiotensin system inhibition: a biomeasure of therapeutic success? Kidney Int. 2011;80(3):235-7.

34. Lewis EF, Claggett B, Parfrey PS, Burdmann EA, McMurray JJ, Solomon SD, et al. Race and ethnicity influences on cardiovascular and renal events in patients with diabetes mellitus. Am Heart J. 2015;170(2):322-9.

35. Lipworth L, Mumma MT, Cavanaugh KL, Edwards TL, Ikizler TA, Tarone RE, et al. Incidence and predictors of end stage renal disease among low-income blacks and whites. PLoS ONE. 2012;7(10): 48407.

36. Heerspink HJL, Stefansson BV, Correa-Rotter R, Chertow GM, Greene T, Hou FF, et al. Dapagliflozin in patients with chronic kidney disease. N Engl J Med. 2020;383(15):1436-46. 